\title{
Mudanças no estilo de vida e fatores de risco para doenças crônicas não transmissíveis e sistema imune de mulheres sedentárias
}

\author{
Lifestyle changes and risk factors for non-communicable \\ chronic diseases and immune system of sedentary women
}

Carlos Alexandre FETT ${ }^{1}$

Waléria Christiane Rezende FETT'1,2

Gilberto João PADOVAN²

Julio Sergio MARCHINI2

RE S U M O

\section{Objetivo}

Observar os efeitos de dois meses de um programa de reeducação de hábitos sobre variáveis do sistema imunológico e de risco para doenças crônicas não transmissíveis e as associações destas entre si e com a composição corporal.

\section{Métodos}

Cinqüenta mulheres sedentárias, com idade de 36 anos ( $D P=10)$, e índice de massa corporal de $31 \mathrm{~kg} / \mathrm{m}^{2}$ ( $\mathrm{DP}=12)$ participaram do estudo. Foram avaliados a composição corporal (antropometria e bioimpedância), o hemograma, o perfil lipídico, as concentrações plasmáticas das vitaminas A, C, betacaroteno, do zinco, do ácido úrico e da glicemia. O treinamento consistia de circuito de peso ou caminhada por $1 \mathrm{~h}$, três vezes por semana no primeiro mês e $1 \mathrm{~h}$, quatro vezes por semana no segundo mês, mais reeducação alimentar.

\section{Resultados}

A gordura corporal, as concentrações plasmáticas do ácido úrico, do colesterol total e da lipoproteína de alta densidade foram significativamente reduzidas. Os triglicérides, a lipoproteína de baixa densidade e as variáveis do sistema imunológico não foram alterados. O zinco não foi associado a qualquer variável. Os glóbulos brancos, os linfócitos, as plaquetas e a vitamina C foram positivamente associados à gordura corporal e negativamente, quando divididos pela massa corporal $(\mathrm{kg})$. O colesterol total e a lipoproteína de baixa densidade divididos pela concentração de vitamina $A$ e do betacaroteno foram negativamente correlacionados à gordura corporal. A vitamina C teve a maior correlação com outros fatores bioquímicos de risco.

\footnotetext{
1 Universidade Federal de Mato Grosso, Faculdade de Educação Física. Av. Fernando Correa da Costa, s/n., Campus Ginásio de Esportes, 78060-900, Cuiabá, MT, Brasil. Correspondência para/Correspondence to: C.A. FEET. E-mail: <cafett@hotmail.com>.

${ }^{2}$ Universidade de São Paulo, Faculdade de Medicina de Ribeirão Preto, Departamento de Clínica Médica, Laboratório de Espectrometria de Massa, Divisão de Nutrologia. Ribeirão Preto, SP, Brasil.
} 
246 | C.A. FETT et al.

\section{Conclusão}

Houve redução dos fatores de risco para doenças crônicas não transmissíveis, mas não nos marcadores do sistema imunológico. O aumento da gordura corporal foi associado negativamente aos marcadores do sistema imunológico e das vitaminas.

Termos de indexação: Composição corporal. Leucócitos. Vitaminas. Zinco.

\section{A B S T R A C T}

\section{Objective}

The objective of this study was to observe the effects of two months of a lifestyle-changing program on the immune system and risk of non-communicable chronic diseases and how they associate with each other and with body composition.

\section{Methods}

Fifty women aged 36 years $(S D=10)$ with a mean body mass index of $31 \mathrm{~kg} / \mathrm{m}^{2}(S D=12)$ participated in this study. The following data were investigated: body composition (by anthropometry and bioimpedance), complete blood count, lipid profile, plasma concentrations of vitamins A and C, beta-carotene, zinc, uric acid and glucose. The program consisted of circuit weight training or walking for 1 hour, three times per week during the first month and for 1 hour, four times per week during the second month and changes to their eating habits.

\section{Results}

There was a significant reduction in body fat and plasma concentrations of uric acid, total cholesterol and high-density lipoprotein. Triglycerides, low density lipoprotein and immune system variables remained unchanged. Zinc was not associated with any variable. The leukocytes, lymphocytes, platelets and vitamin C were positively associated with body fat and negatively associated when divided by body mass (kg). Total cholesterol and low-density lipoprotein divided by vitamin A and beta-carotene concentrations had a negative correlation with body fat. Vitamin C correlated the most with other biochemical risk factors.

\section{Conclusion}

The risk factors for non-communicable chronic diseases reduced, but immune system markers remained unchanged. Increased body fat was negatively associated with immune system markers and vitamins.

Indexing terms: Body. Leukocytes. Vitamins. Zinc.

\section{N T R O D U Ç Ã O}

Existem em torno de 800 milhões de indivíduos mal nutridos em todo o mundo, especialmente mulheres e crianças. As vitaminas, os minerais e os elementos traços são principalmente depletados do organismo devido à poluição do meio ambiente pela ação do homem. Elementos tóxicos como chumbo, arsênico, mercúrio e outros metais pesados entram na cadeia alimentar e reduzem os estoques orgânicos, principalmente os das vitaminas A e $C$, e dos minerais ferro, iodo, cálcio, ácido fólico e zinco (Zn), decrescendo a atividade imunológica ${ }^{1}$

O bom funcionamento do sistema imunológico é dependente de vários nutrientes e entre eles estão alguns micronutrientes que têm papel chave no organismo. Sua deficiência leva ao aumento do estresse oxidativo e à depressão do sistema imunológico ${ }^{2}$. Várias condições, como o aumento da gordura corporal e o excesso de atividade física, podem expor o organismo ao aumento do estresse oxidativo ${ }^{3,4}$. Entretanto, o processo adaptativo ao estímulo causado pela atividade física crônica aumenta as enzimas antioxidantes reduzindo os efeitos das lesões causadas pelos radicais livres ${ }^{3}$.

A inflamação crônica indicada por uma alta contagem dos glóbulos brancos está associada a fatores de risco presentes na obesidade, no diabetes tipo 2 e na síndrome metabólica ${ }^{5}$. Os 
triglicérides e a glicose estão positivamente associadas aos glóbulos brancos ${ }^{5}$; já o aumento do colesterol total, da lipoproteína de baixa densidade (LDL), dos triglicérides e a redução da lipoproteína de alta densidade (HDL), estão associados à piora do sistema antioxidante. Por exemplo, a aterosclerose é um processo que sofre influência da interação entre lipídios e antioxidantes. A aterosclerose não depende apenas do aumento da LDL, mas sim de que esta seja oxidada e catabolizada pelos macrófagos levando à formação de células espumosas (foam cells), sendo este o primeiro passo para lesão na aterogênese, tendo efeitos citotóxicos e quimiotáticos ${ }^{6}$. Existem vários mecanismos de proteção para a formação dos radicais livres que podem oxidar a LDL, particularmente as enzimas antioxidantes endógenas, as vitaminas antioxidantes e outros antioxidantes dietéticos ${ }^{2,4}$.

O zinco é um micronutriente envolvido nas vias metabólicas da síntese de proteínas, no metabolismo dos carboidratos, dos lipídios e dos ácidos nucléicos. Está relacionado à melhora da sensibilidade à insulina e à redução da gordura corporal, podendo assim favorecer tanto aos obesos quanto aos diabéticos do tipo 2. Os obesos têm demonstrado aportes reduzidos deste mineral e quando repostos por suplementação apresentam melhora da sensibilidade à insulina. A deficiência de Zn em indivíduos obesos, quando comparada a indivíduos normais, estava associada também à deficiência da glutationa peroxidase dos eritrócitos, da superóxido dismutase e da cobre-zinco, prejudicando o status antioxidante, que talvez, tenham implicações no desenvolvimento da obesidade, do diabetes tipo 2 e das doenças cardiovasculares ${ }^{4}$.

A atividade física per se aumenta a produção de radicais livres e, embora o sistema antioxidante endógeno seja adaptado ao exercício crônico ${ }^{3}$, atividades aeróbias de longa duração são associadas ao catabolismo das gorduras e parecem necessitar de maior aporte exógeno de vitaminas antioxidantes, como o betacaroteno, as vitaminas C e E, para evitar a exacerbação do estresse oxidativo ${ }^{7}$. Por outro lado, normalmente é recomendado que obesos façam atividades físicas aeróbias para a redução da gordura corporal ${ }^{8}$. Ainda, é relatado que indivíduos obesos apresentam deficiência destas vitaminas antioxidantes e, associados ao exercício físico, poderiam aumentar a peroxidação lipidica9 ${ }^{9}$. Nesse sentido, obesos seguindo esta orientação, poderiam expor o sistema imunológico a uma reação inflamatória crônica ${ }^{2,3,5,6}$.

Portanto, os objetivos deste estudo foram verificar em mulheres adultas sedentárias: 1) os efeitos de um programa de reeducação de hábitos (exercício físico e dieta), sobre variáveis diretas e indiretas do sistema imunológico; 2) a influência deste programa sobre a composição corporal e os marcadores bioquímicos sangüíneos de risco para doenças crônicas não transmissíveis (DCNT); e, 3) as associações entre os marcadores do sistema imunológico, da gordura corporal e dos marcadores de risco para DCNT.

\section{MÉ T O D O S}

Foram distribuídos cartazes na Universidade de São Paulo (USP), campus de Ribeirão Preto, convidando mulheres interessadas em participar do presente estudo. O cartaz continha, informações para orientar sobre o cálculo do índice de massa corporal (IMC, $\mathrm{kg} / \mathrm{m}^{2}$ ), explicitando ser necessário ter $I M C>20$, o telefone para contato com a equipe, o local e o horário da primeira reunião com as interessadas. Entraram em contato com a equipe 110 mulheres; 72 preenchiam os critérios de inclusão e 50 mulheres sedentárias (menos de duas sessões semanais de atividade física formal no último semestre) entre 18 e 55 anos (média - $\mathrm{M}=36$ anos, desvio-padrão - $\mathrm{DP}=10$ ), com índice de massa corporal de 23 a $57 \mathrm{~kg} / \mathrm{m}^{2}$ $\left(\mathrm{IMC}=31 \mathrm{~kg} / \mathrm{m}^{2}, \mathrm{DP}=12\right)$, concluíram todos os exames e os testes propostos. Este largo espectro do IMC foi para observar sua influência sobre as variáveis bioquímicas estudadas. A hipótese era que seu aumento estaria positivamente correlacionado à piora dos marcadores do sistema imunológico e que poderiam ser melhorados com a redução do peso corporal. A amplitude da idade 
também foi grande, a fim de verificar se com seu avanço haveria comprometimento de variáveis do sistema imunológico.

O exame clínico foi realizado por médico da equipe de Nutrologia do Departamento de Clínica Médica da Faculdade de Medicina de Ribeirão Preto da Universidade de São Paulo (FMRP - USP). As voluntárias assinaram documento de consentimento livre e esclarecido aprovado pelo Comitê de Ética em Pesquisa do Hospital das Clínicas da FMRP - USP (HCFMRP - USP; processo HCRP n ${ }^{\circ}$ 5317/2002).

Foram excluídas as mulheres que apresentavam histórico de doenças metabólicas, além da obesidade em si, as fumantes, as etilistas ( $>15 \mathrm{~g}$ equivalentes de etanol/dia); as usuárias de medicamentos como betas bloqueadores e drogas simpatomiméticas.

A massa corporal $(\mathrm{kg})$ foi obtida utilizando-se uma balança de plataforma, da marca Filizola ${ }^{\circledR}$ Eletrônica ID 1500 (São Paulo: SP, Brasil), com precisão de $0,1 \mathrm{~kg}$ e a estatura com uma barra metálica graduada com precisão de $0,5 \mathrm{~cm}$.

Para a medida da espessura das pregas cutâneas foi utilizado um adipômetro da marca Lange ${ }^{\circledR}$ (Beta Technology INC; Santa Cruz: CA, EUA), com pressão constante de $10 \mathrm{~g} / \mathrm{mm}^{2}$ na superfície de contato, precisão de $1 \mathrm{~mm}$ e escala de $0-65 \mathrm{~mm}$. O valor anotado foi a média de três medidas consecutivas. As pregas cutâneas mensuradas foram: tríceps, supra-ilíaca e coxa. A densidade corporal foi estimada a partir das medidas das pregas cutâneas corrigidas pela idade e então convertida para percentual de gordura corporal ${ }^{8}$.

Foi medida a circunferência do abdômen sobre a cicatriz umbilical, paralelamente ao solo, com uma fita metálica, inextensível e flexível.

A composição corporal também foi estimada pela bioimpedância (Quantum BIA-101Q ${ }^{\circledR}$, Serial n Q 1559; RJL Systems, Inc. Clinton: MI, EUA), utilizando a fórmula validada por Segal et al. ${ }^{10}$ : MMbia $=0,0011 E^{2}-0,021 R+0,232 P-0,0681=14,595$, sendo: $\mathrm{MMbia}=$ massa magra obtida pela bioim- pedância em kg; E = Estatura em m; R = Resistência em $\Omega ; \mathrm{P}=$ Peso em $\mathrm{kg} ; \mathrm{I}=$ Idade em anos.

O sangue foi coletado seguindo a rotina de atendimento dos pacientes obesos do HCFMRP - USP, em tubo contendo EDTA e realizado o hemograma completo. Os triglicérides, HDL e colesterol total foram dosados por métodos enzimáticos seguindo as orientações dos manuais dos cartuchos de reagentes Flex (Flex ${ }^{\circledast}$ Reagent Cartridge, Dade Behring Inc, 2001, 2002, e 2003, respectivamente). A LDL foi calculada a partir da seguinte fórmula: $L D L=C T-H D L-T g / 5$. A glicemia foi dosada pela adaptação do método da Hexokinase-glicose-6-fosfato. As vitaminas A, C e o betacaroteno foram dosados no laboratório de nutrição, do HCFMRPUSP e seguiram as orientações do Manual de Procedimentos Técnicos ${ }^{11}$.

O zinco foi dosado no Laboratório de Espectrometria de Massa da FMRPUSP, pelo método de absorção atômica, usando o modo chama com acetileno super puro, fluxo de dois L/min do comburente e o ar sintético de oito L/min, com spray time de sete segundos, tempo de integração de cinco segundos, usando-se, uma curva padrão, com as seguintes concentrações expressas em $\mathrm{mg} / \mathrm{L}: 0,0 ; 0,05 ; 0,10 ; 0,20 ; 0,40$; condições da lâmpada: wave lenght $=213,9 \mathrm{~nm}$; slidt width $=0,7 \mathrm{~nm}$; corrente $=8 \mathrm{~mA}$.

As voluntárias foram distribuídas aleatoriamente em um treinamento em circuito ou caminhada, equalizados na intensidade pela freqüência cardíaca (65-75\% da máxima), três vezes por semana no primeiro mês e quatro vezes por semana no segundo mês. O protocolo de treinamento em circuito era composto por 15 estações de exercícios resistidos, feitos em um salão com um tatame, com 30 segundos de duração por estação, intercalados com 30 segundos de caminhada. $\mathrm{O}$ grupo caminhada realizava somente esta atividade de forma contínua. As sessões eram compostas de 10 minutos de aquecimento, 40 minutos de parte principal da aula e 10 minutos de volta à calma.

Todas as participantes foram orientadas quanto à reeducação alimentar e à mudança de 
estilo de vida, em reuniões realizadas semanalmente com a seguinte equipe: uma médica, uma nutricionista e dois profissionais de educação física.

A fim de verificar a associação do programa de reeducação de hábitos a outras variáveis e estas entre si, os grupos foram tratados como um só. Os resultados estão apresentados sob forma de média e desvio-padrão. Devido à perda de voluntárias, o conjunto de dados iniciais (M1), foi comparado ao conjunto de dados finais (M2) do estudo, pelo teste $t$ não pareado. A associação entre duas variáveis foi obtida pela correlação linear de Pearson. Todas as conclusões estatísticas foram realizadas considerando-se o nível de 5\% de significância com intervalo de confiança de $95 \%$.

\section{RESULTADOS}

Todas as medidas da gordura corporal, concentrações plasmáticas do ácido úrico, do colesterol total e do HDL foram significantemente reduzidas. Os triglicérides apenas tenderam à redução. O LDL e todas as variáveis diretas ou indiretamente ligadas ao sistema imunológico não foram alterados pelos dois meses de programação, nem mesmo quando corrigidos pela massa corporal (kg) (Tabela 1).

Para verificar a classificação de diferentes variáveis nos dois momentos foram comparadas as médias do M1 e do M2 com os valores de corte de referência (deficiência, normalidade, excesso), que aparecem entre parênteses.

No M1 as voluntárias eram classificadas como obesas (IMC $>30 \mathrm{~kg} / \mathrm{m}^{2}$ ) e no M2 como sobrepeso $\left(I M C=25-29,9 \mathrm{~kg} / \mathrm{m}^{2}\right)^{12}$. Quanto à circunferência do abdômen elas eram classificadas no $\mathrm{M} 1$ como zona de risco $(>88 \mathrm{~cm})$; houve redução significante desta variável, mas continuaram classificadas como zona de risco no $\mathrm{M} 2^{13}$. A porcentagem de gordura estimada pelos dois métodos também foi reduzida significantemente, mas

Tabela 1. Perfil de mulheres adultas sedentárias antes (M1) e depois (M2) de dois meses de um programa de reeducação de hábitos. Ribeirão Preto (SP), 2007

\begin{tabular}{|c|c|c|c|c|c|c|c|}
\hline \multirow{2}{*}{ Variáveis/momentos } & \multicolumn{2}{|c|}{ M1 } & \multirow{2}{*}{$\mathrm{n}$} & \multicolumn{2}{|c|}{ M2 } & \multirow{2}{*}{$\mathrm{n}$} & \multirow{2}{*}{$p$} \\
\hline & M & DP & & M & DP & & \\
\hline Índice de Massa Corporal (IMC, kg/m²) & 31,0 & 6,0 & 49 & 29,0 & 6,0 & 27 & 0,0001 \\
\hline Circunferência do abdômen $(\mathrm{cm})$ & 103,0 & 14,0 & 50 & 99,0 & 16,0 & 27 & 0,0001 \\
\hline Gordura corporal \% (bioimpedância) & 40,0 & 6,0 & 45 & 37,0 & 5,0 & 15 & 0,0001 \\
\hline Gordura corporal \% (antropometria) & 42,0 & 6,0 & 50 & 36,0 & 6,0 & 27 & 0,0002 \\
\hline Glóbulos brancos $\left(\times 10^{3} / \mathrm{mm}^{3}\right)$ & 7,0 & 1,8 & 22 & 6,8 & 1,7 & 22 & $>0,05$ \\
\hline Linfócitos (\%) & 22,0 & 5,0 & 22 & 22,0 & 5,0 & 22 & $>0,05$ \\
\hline Plaquetas $\left(\times 10^{3} / \mathrm{mm}^{3}\right)$ & 269,0 & 44,0 & 22 & 281,0 & 56,0 & 22 & $>0,05$ \\
\hline Ácido úrico $(\mathrm{mg} / \mathrm{dL})$ & 5,0 & 1,0 & 49 & 4,5 & 1,0 & 22 & 0,0094 \\
\hline Colesterol total (mg/dL) & 184,0 & 29,0 & 48 & 179,0 & 27,0 & 22 & 0,0193 \\
\hline Triglicérides (mg/dL) & 105,0 & 52,0 & 48 & 87,0 & 35,0 & 22 & 0,0640 \\
\hline $\mathrm{LDL}(\mathrm{mg} / \mathrm{dL})$ & 107,0 & 27,0 & 48 & 105,0 & 18,0 & 19 & $>0,05$ \\
\hline $\mathrm{HDL}(\mathrm{mg} / \mathrm{dL})$ & 55,0 & 12,0 & 48 & 52,0 & 11,0 & 19 & 0,0187 \\
\hline Glicemia (mg/dL) & 93,0 & 10,0 & 46 & 89,0 & 6,0 & 22 & $>0,05$ \\
\hline Zinco $(\mathrm{mg} / \mathrm{dL})$ & 0,4 & 0,02 & 34 & 0,4 & 0,03 & 25 & $>0,05$ \\
\hline Vitamina A ( $\mu \mathrm{g} / \mathrm{dL})$ & 49,0 & 14,0 & 27 & 50,0 & 18,0 & 5 & $>0,05$ \\
\hline Vitamina C (mg/dL) & 0,4 & 0,06 & 27 & 0,4 & 0,05 & 5 & $>0,05$ \\
\hline Betacaroteno $(\mu \mathrm{g} / \mathrm{dL})$ & 194,0 & 62,0 & 27 & 170,0 & 46,0 & 5 & $>0,05$ \\
\hline $\mathrm{CT} / \mathrm{HDL}$ & 3,5 & 0,92 & 48 & 3,5 & 86,0 & 19 & $>0,05$ \\
\hline $\mathrm{Zn} / \mathrm{kg}$ & 0,005 & 0 & 34 & 0,006 & 0 & 23 & $>0,05$ \\
\hline Glóbulos brancos/kg & 81,0 & 25,0 & 49 & 91,0 & 23,0 & 26 & $>0,05$ \\
\hline Linfócitos/kg & 26,0 & 5,0 & 47 & 28,0 & 5,0 & 22 & $>0,05$ \\
\hline
\end{tabular}

Teste $t$ não pareado. Valores expressos em média (M) e desvio-padrão (DP). 
permaneceu dentro da faixa considerada como excesso de gordura (> $23 \%$ para mulheres) ${ }^{8}$ (Tabela 1).

Os glóbulos brancos (5 000 a 10 000/mm³) e as plaquetas ( 150.000 a $350000 / \mathrm{mm}^{3}$ ) estavam dentro dos valores de normalidade, mas os linfócitos (25 a 33\%) estavam abaixo dos valores de normalidade ${ }^{11}$, em ambos os momentos (Tabela 1).

O ácido úrico (2,4 a 7,0mg/dL), o colesterol total $(<200 \mathrm{mg} / \mathrm{dL})$, a $\mathrm{LDL}(<130 \mathrm{mg} / \mathrm{dL})$, os triglicérides ( $<200 \mathrm{mg} / \mathrm{dL})$, o HDL $(\geq 35 \mathrm{mg} / \mathrm{dL})$ e a glicemia (70,0 a 110,0mg/dL), foram reduzidos significantemente; estavam no M1 em valores considerados desejáveis e permaneceram assim no $\mathrm{M}^{11}$ (Tabela 1).

No M1 a vitamina A estava próxima aos valores considerados aumentados $(\geq 50 \mu \mathrm{g} / \mathrm{dL})^{11}$; a vitamina $C$ era considerada deficiente $(<0,4 \mathrm{mg} / \mathrm{dL})^{14}$; o betacaroteno $(\geq 100 \mu \mathrm{g} / \mathrm{dL})^{11}$ e o zinco $(\geq 0,1 \mathrm{mg} / \mathrm{dL})^{15}$ estavam com os valores considerados aumentados; todas permaneceram sem alterações no M2 (Tabela 1).

Foi feita a correlação linear de Pearson do IMC, da circunferência do abdômen, da porcentagem de gordura pela bioimpedância e pela antropometria e da idade a variáveis ligadas direta ou indiretamente ao sistema imunológico. As concentrações plasmáticas de zinco, das vitaminas $A$, do betacaroteno, das plaquetas, das razões $\mathrm{Zn} / \mathrm{kg}$, colesterol total/Zn, LDL/Zn, HDL/Zn, triglicérides/Zn, colesterol total/Vitamina $A$ LDLNitamina A, HDL/Nitamina A, triglicérides/ Vitamina A, colesterol total/Vitamina C, LDLNitamina C, triglicérides/Vitamina C, HDL/betacaroteno, triglicérides/betacaroteno e linfócitos $/ \mathrm{kg}$ não foram correlacionadas a medida alguma da gordura corporal $(P>0,05)$. As correlações deste tópico que tiveram alguma significância estão na Tabela 2.

Vários fatores bioquímicos foram correlacionados entre si. Os glóbulos brancos correlacionaram-se a um fator, os linfócitos a três, a vitamina $\mathrm{C}$ a cinco, o ácido úrico a dois, a vitamina $A$ a dois, o betacaroteno a dois, o colesterol total a seis, entretanto, três destes são fatores ligados diretamente aos lipídios circulantes, sendo esperado que fossem correlacionados; o HDL a três, os triglicérides a cinco, entretanto, três destes são fatores ligados diretamente aos lipídios circulantes, sendo esperado que fossem correlacionados; a fração CT/HDL a seis, entretanto, três destes são fatores ligados diretamente aos lipídios circulantes, sendo esperado que fossem correlacionados; os linfócitos $/ \mathrm{kg}$ foram correlacionados a um fator bioquímico (Tabela 3).

Tabela 2. Associação entre variáveis relacionadas à gordura corporal e marcadores bioquímicos sangüíneos e várias razões de mulheres adultas sedentárias. Ribeirão Preto (SP), 2007.

\begin{tabular}{|c|c|c|c|c|c|}
\hline Variáveis & $\mathrm{IMC}\left(\mathrm{kg} / \mathrm{m}^{2}\right)$ & $\mathrm{CAb}(\mathrm{cm})$ & $\%$ G Bia & $\%$ G Antro & Idade (anos) \\
\hline Glóbulos brancos $\left(\times 10^{3} / \mathrm{mm}^{3}\right)$ & 0,$52 ; 0,27 \dagger$ & $0,47 ; 0,22\rfloor$ & $>0,05$ & 0,$25 ; 0,06$ & $-0,31 ; 0,10^{*}$ \\
\hline Glóbulos brancos/kg & $-0,28 ; 0,08^{*}$ & $-0,30 ; 0,10$ * & $-0,44 ; 0,19\rfloor$ & $-0,38 ; 0,14^{*}$ & $>0,05$ \\
\hline Linfócitos (\%) & 0,$53 ; 0,28 \ddagger$ & 0,$53 ; 0,28 \neq$ & 0,$37 ; 0,14^{*}$ & 0,$29 ; 0,09 *$ & $>0,05$ \\
\hline Plaquetas $\left(\times 10^{3} / \mathrm{mm}^{3}\right)$ & $>0,05$ & $>0,05$ & $>0,05$ & $0,40 ; 0,16\rfloor$ & $>0,05$ \\
\hline Plaquetas/kg & لـ, & $-0.39 ; 16\rfloor$ & $-0,33 ; 11 *$ & $>0,05$ & $>0,05$ \\
\hline Vitamina $C(\mathrm{mg} / \mathrm{dL})$ & 0,$48 ; 0,23^{*}$ & 0,$41 ; 0,17^{*}$ & $>0,05$ & $>0,05$ & $>0,05$ \\
\hline Vitamina $\mathrm{C} / \mathrm{kg}$ & $-0,51 ; 0,26^{*}$ & $-0,50 ; 0,25^{*}$ & $-0,44 ; 0,20 *$ & $-0,57 ; 0,32\rfloor$ & $>0,05$ \\
\hline Vitamina $\mathrm{A} / \mathrm{kg}$ & $-0,50 ; 0,24^{*}$ & $-0,45 ; 0,20^{*}$ & $>0,05$ & $-0,45 ; 0,20 *$ & 0,$51 ; 0,26^{*}$ \\
\hline Betacaroteno/kg & $-0,52 ; 0,27^{*}$ & $-0,50 ; 0,25^{*}$ & $>0,05$ & $-0,57 ; 0,32$ لـ & $>0,05$ \\
\hline CT/betacaroteno & $>0,05$ & 0,$42 ; 0,18^{*}$ & $>0,05$ & 0,$44 ; 0,19^{*}$ & $>0,05$ \\
\hline LDL/betacaroteno & $>0,05$ & 0,$48 ; 0,23 *$ & $>0,05$ & 0,$45 ; 0,20 *$ & $>0,05$ \\
\hline
\end{tabular}

Correlação linear de Pearson $\left(r ; r^{2}\right) ; n=48 ;$ para dosagens de vitaminas, $n=27 .{ }^{*}=p<0,05 ; \exists=p<0,005 ; \quad t=p<0,0005 ; \neq=p<0,0001$;

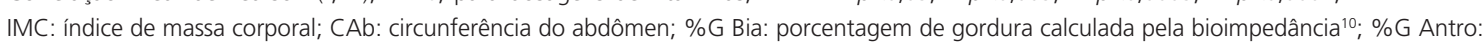
porcentagem de gordura calculada pela antropometria ${ }^{8}$. 
REEDUCAÇÃO DE HÁBITOS E SISTEMA IMUNOLÓGICO | 251

Tabela 3. Associação entre vários fatores bioquímicos sangüíneos de mulheres adultas sedentárias. Ribeirão Preto (SP), 2007.

\begin{tabular}{|c|c|c|c|c|c|c|c|c|c|c|c|c|c|}
\hline V & $\mathrm{GB}$ & L & P & $\mathrm{Zn}$ & VA & VC & $\beta$ & $A U$ & $\mathrm{CT}$ & LDL & $\mathrm{HDL}$ & $\mathrm{Tg}$ & $\mathrm{F}$ \\
\hline $\mathrm{L}$ & $0,56 \neq$ & & & & & & & & & & & & \\
\hline$P$ & $0,33^{*}$ & $\neq$ & & & & & & & & & & & \\
\hline $\mathrm{Zn}$ & $\neq$ & $\neq$ & $\neq$ & & & & & & & & & & \\
\hline VA & $\neq$ & $\neq$ & $\neq$ & $\neq$ & & & & & & & & & \\
\hline VC & $0,39 *$ & $0,66 \dagger$ & $\neq$ & $\neq$ & $\neq$ & & & & & & & & \\
\hline$\beta$ & $\neq$ & $\neq$ & $\neq$ & $\neq$ & $\neq$ & $\neq$ & & & & & & & \\
\hline$A U$ & $\neq$ & $0,30^{*}$ & $\neq$ & $\neq$ & $\neq$ & $\neq$ & 0,41 * & & & & & & \\
\hline$C T$ & $\neq$ & $\neq$ & $\neq$ & $\neq$ & $0,70+$ & $0,53 ¥$ & $\neq$ & $0,32^{*}$ & & & & & \\
\hline LDL & $\neq$ & $\neq$ & $\neq$ & $\neq$ & $0,62 ¥$ & $\neq$ & $0,42^{*}$ & $0,38^{*}$ & $0,89+$ & & & & \\
\hline $\mathrm{HDL}$ & $\neq$ & $\neq$ & $\neq$ & $\neq$ & $\neq$ & $\neq$ & $\neq$ & $\neq$ & $\neq$ & $\ddagger$ & & & \\
\hline $\operatorname{Tg}$ & $\neq$ & $0,35^{*}$ & $\neq$ & $\neq$ & $\neq$ & $0,45^{*}$ & $\neq$ & $\neq$ & $0,36^{*}$ & $\neq$ & $-0,30$ * & & \\
\hline$F$ & $\neq$ & $\neq$ & $\neq$ & $\neq$ & $\neq$ & $\neq$ & $\neq$ & $0,44 ¥$ & $0,49+$ & $0,66+$ & $-0,77 \dagger$ & $0,54 \dagger$ & \\
\hline $\mathrm{Gl}$ & $\neq$ & $\neq$ & $\neq$ & $\neq$ & $\neq$ & $\neq$ & $\neq$ & $\neq$ & $\neq$ & $\neq$ & $-0,31$ * & $\neq$ & 0,31 * \\
\hline $\mathrm{GB} / \mathrm{kg}$ & - & - & $\ddagger$ & $\neq$ & $\neq$ & $\neq$ & $\neq$ & $\neq$ & $\neq$ & $\neq$ & $\neq$ & $\neq$ & $\neq$ \\
\hline $\mathrm{L} / \mathrm{kg}$ & - & - & $\neq$ & $\neq$ & $\neq$ & $0,59 ¥$ & $\neq$ & $\neq$ & $\neq$ & $\neq$ & $\neq$ & $\neq$ & $\neq$ \\
\hline
\end{tabular}

Correlação linear de Pearson (r); $n=48$; para as dosagens de vitaminas o $n=27$. Estatística: $\neq p>0,05 ;{ }^{*}=p<0,05 ; ¥=p<0,005 ; \dagger=p<0,0005$. V: variáveis; GB: glóbulos brancos; P: plaquetas (x103/mm³); L: linfócitos (\%); VA: vitamina A e $\beta$ : betacaroteno ( $\mu \mathrm{g} / \mathrm{dL})$; Zn: Zinco; VC: vitamina C; AU: ácido úrico; CT: colesterol total; LDL: lipoproteína de baixa densidade; HDL: lipoproteína de alta densidade; Tg: triglicérides; Gl: glicose (mg/dL); F: fração CT/HDL.

\section{I S C U S S Ã O}

O presente estudo demonstrou que dois meses de um programa de reeducação de hábitos, incluindo exercício físico e orientação nutricional, reduziram vários fatores de risco relacionados a doenças metabólicas, sendo que a composição corporal e o perfil lipídico foram positivamente modificados. Entretanto, as variáveis associadas direta ou indiretamente ao sistema imunológico não foram afetadas pela redução do peso corporal e pelo exercício físico.

A exacerbação da oxidação das gorduras pelo exercício físico pode deprimir o sistema imunológico ${ }^{3}$. No caso do presente estudo, as atividades visavam ao aumento da oxidação das gorduras e estas mulheres apresentavam deficiência de vitamina $C$ e linfócitos no momento inicial, podendo expô-las a depressão do sistema imunológico, o que não foi observado. Por outro lado, as outras variáveis do sistema imunológico, vitaminas e zinco estavam em concentrações normais e não foram impactadas pelo programa de reeducação de hábitos. Ainda, a exacerbação na produção de radicais livres é evidente em indivíduos com treinamento de alto volume e inten- sidade ${ }^{7}$. O fato de a atividade física praticada estar em um nível moderado e haver a contribuição da reeducação alimentar, com orientação para o aumento do consumo de fibras, frutas e verduras ${ }^{16}$, podem ter contribuído para manter o status do sistema imunológico.

Quanto às associações, vários marcadores bioquímicos sangüíneos correlacionavam-se entre si, mas sem muita consistência e, de forma geral, as medidas da gordura corporal afetavam negativamente as variáveis associadas ao sistema imunológico. Das medidas da gordura corporal a que mais se associou negativamente às variáveis do sistema imunológico foi a circunferência do abdômen, seguida pelo percentual da gordura corporal estimado pela antropometria e pelo IMC. Já, o percentual de gordura estimado pela bioimpedância não teve correlação com a maioria das variáveis comparadas. Outros autores, também observaram que as medidas antropométricas eram melhor associadas ao risco de desenvolver aterosclerose, do que as medidas da bioimpedância e, como aqui, observaram que a circunferência do abdômen era a que apresentava maior associação ${ }^{17}$. 
Tem sido demonstrado que a suplementação de antioxidantes pode reduzir o estresse oxidativo ${ }^{18}$. No presente estudo, embora não tenha havido o uso de suplementos, a vitamina $C$ foi a que maior número de correlações positivas teve com outras variáveis bioquímicas, reforçando sua importância como imunomoduladora. Esta vitamina e os leucócitos rapidamente declinam durante as infecções e o estresse. Outros observaram que o aumento da concentração plasmática de vitamina $C$ era associado à melhora dos componentes do sistema imunológico, tais como, a atividade antimicrobiana e das células natural killer, a proliferação de linfócitos e a quimiotaxia ${ }^{19}$. A vitamina $C$ e os linfócitos destas mulheres estavam em concentrações consideradas deficientes, o que poderia contribuir para piora das defesas imunológicas e da neutralização da produção de radicais livres. Isto pode ser devido ao fato de muitas delas no início do estudo fazerem uso de uma dieta do tipo cafeteria, consumindo poucos vegetais, e, portanto, possibilitando o déficit de vitamina $C$, não ocorrendo o mesmo com as vitaminas lipossolúveis, mais disponíveis neste tipo de dieta. Ainda, a vitamina $\mathrm{C}$ foi associada positivamente aos glóbulos brancos, aos linfócitos e aos linfócitos $/ \mathrm{kg}$, sugerindo ser importante na modulação do sistema imunológico destas mulheres.

Por outro lado, a vitamina C e os glóbulos brancos foram positivamente correlacionados às variáveis da gordura corporal, sugerindo em uma primeira leitura, estarem mais disponíveis com o aumento da obesidade. Entretanto, quando corrigidos pelo peso corporal, apresentaram correlação significantemente inversa aos marcadores da gordura corporal, sugerindo que o aumento de gordura neste grupo era prejudicial ao sistema imunológico, corroborando o encontrado por outros pesquisadores ${ }^{4}$. Ainda, indivíduos asmáticos apresentaram níveis mais baixos de vitamina $\mathrm{C}$ e de linfócitos plasmáticos, quando comparados aos indivíduos normais ${ }^{14}$, sugerindo que ambos estão associados e a diminuição da vitamina $C$ prejudica o sistema imunológico. Por outro lado, não foi observada melhora do perfil lipídico e da sensibilidade à insulina, em obesos suplementados com 1g de vitamina $\mathrm{C}$ ao dia, por quatro semanas ${ }^{20}$, sugerindo neste caso, outras interações além destas. Estas controvérsias podem ser explicadas por se tratarem de diferentes populações e hábitos de vida que contribuem com fatores de confusão para as análises.

O betacaroteno e a vitamina $A$, quando corrigidos para massa corporal, também foram negativamente associados à gordura corporal. Estas vitaminas estavam associadas à maioria dos lipídios circulantes, o que é justificado por serem lipossolúveis. Concordando com estas observações, foi verificado em mulheres que as concentrações de betacaroteno sérico eram positivamente associadas à concentração sérica de colesterol, à ingestão de fibras, à ingestão de betacaroteno e negativamente associadas ao fumo e a todas as medidas de obesidade ${ }^{16}$. Benabdeslam et al..$^{21}$ observaram que as concentrações do retinol, do alfacaroteno e do betacaroteno, em especial deste último, eram todas reduzidas em pacientes com fibrose cística, comparadas ao controle e que esses pacientes estavam mais expostos ao estresse oxidativo, estimado por biomarcadores da peroxidação lipídica. Portanto, parece que no presente estudo, estas vitaminas estavam proporcionalmente reduzidas em relação ao aumento da gordura corporal, o que poderia contribuir para o aumento do estresse oxidativo.

Foi demonstrado que os níveis das vitaminas $\mathrm{A}, \mathrm{E}, \mathrm{C}$ e de ácido úrico têm influência limitada, mas significante na proteção do DNA ${ }^{22}$, e que a vitamina $A$, E e o selênio são negativamente relacionados ao estresse oxidativo e aos problemas de saúde ${ }^{2}$. Não foi avaliado diretamente o estresse oxidativo, portanto, não é possível fazer esta correlação no presente estudo. Todavia, as contagens de glóbulos brancos, de linfócitos e de linfócitos $/ \mathrm{kg}$, foram positivamente correlacionadas à concentração plasmática de vitamina C. Isto sugere influência positiva deste micronutriente sobre o sistema imunológico. Entretanto, as outras vitaminas e o zinco não apresentaram esta associação, corroborando a observação de Foksinki et al. ${ }^{22}$, de que os níveis destes micronutrientes flutuam em relação ao sistema imunológico. 
Ainda, o aumento da contagem de glóbulos brancos é associado a inflamações crônicas, típicas da obesidade, e este aumento está positivamente associado às concentrações de triglicérides e de glicose $\mathrm{e}^{5}$. Mas, no presente estudo, estas associações não foram observadas, possivelmente pelo fato de os glóbulos brancos se encontrarem em valores considerados normais.

Por outro lado, o presente estudo observou que os linfócitos foram associados também ao ácido úrico e aos triglicérides. $\mathrm{O}$ ácido úrico tem função antioxidante, mas quando aumentado é considerado um fator de risco para DCNT ${ }^{23}$. Talvez por causa destas características o ácido úrico foi associado à vitamina $\mathrm{C}$ e ao betacaroteno, mas também ao colesterol total e ao LDL, constituindo, nestes dois últimos casos, um fator de risco para DCNT. Diabéticos tipo 1 fumantes, quando suplementados com ácido úrico $(1000 \mathrm{mg} / \mathrm{d})$ e vitamina C $(1000 \mathrm{mg} / \mathrm{d})$, tinham suas funções endoteliais reestabelecidas ${ }^{24}$, sugerindo que, pelo menos, até certo nível, o ácido úrico é necessário como antioxidante.

Têm sido encontrados valores de zinco mais baixos em indivíduos obesos do que em eutróficos e esta concentração é inversa ao grau de obesidade $^{25}$. Todavia, não foi observado este resultado no presente estudo, sendo que o zinco não se correlacionou à variável alguma da gordura corporal. Pode ser que o fato de nenhuma voluntária ter apresentado deficiência deste mineral, sendo a média inicial e final entre três e quatro vezes o valor considerado normal ${ }^{15}$, tenha afetado a potência estatística.

Também não foi observada relação alguma do zinco com outros fatores de risco bioquímicos sanguíneos. Outros autores também não observaram a relação deste mineral com o perfil lipídico ${ }^{26}$. Estudos ecológicos têm investigado a ingestão de zinco ou o seu status orgânico e a incidência de doenças coronarianas e revelam um padrão não consistente. As observações conflitantes podem ser explicadas pelas diferenças na extensão das doenças cardiovasculares, nos sítios da aterogênese ou por fatores de confusão ${ }^{27}$.
A contagem das plaquetas foi significantemente associada ao percentual de gordura estimado pela antropometria, mas não às outras variáveis da gordura corporal, sugerindo frágil correlação. A atividade plaquetária está associada ao desenvolvimento das placas de ateroma ${ }^{28}$. Foi observado que a atividade plaquetária não estava relacionada ao peso corporal e não apresentava correlação com o excesso de gordura corporal total, mas estava significantemente associada à obesidade abdominal ${ }^{28}$. Não foi medida a atividade plaquetária, mas o número de plaquetas não foi correlacionado à circunferência do abdômen no presente estudo. Entretanto, quando corrigidas pelo peso corporal as plaquetas foram negativamente correlacionadas a três variáveis da gordura corporal, sugerindo que quanto mais gordura corporal nestas mulheres, menor proporção de plaquetas. Talvez, mais importante do que a contagem total seja a ativação das plaquetas por hormônios que estão aumentados na obesidade, como a leptina, que estimula a agregação e a adesão plaquetária ${ }^{29}$. Todavia, não foi comparada esta relação no presente estudo, pois não foi dosada a leptina e ainda, a contagem das plaquetas destas mulheres estava dentro dos valores de normalidade.

O perfil lipídico apresenta associação negativa ao sistema antioxidante ${ }^{6}$. Portanto, para avaliar a proporcionalidade de antioxidantes em relação ao perfil lipídico e ao sistema imunológico, no presente estudo, foram divididas as concentrações de lipídios circulantes, glóbulos brancos e linfócitos pelas concentrações de vitaminas e zinco. A correlação entre a maioria destes resultados e as variáveis da gordura corporal não demonstrou associação. Possivelmente o fato de terem valores de normais a elevados para a maioria das vitaminas e do zinco, contribuiu para este resultado. Entretanto, as relações CT/betacaroteno e LDL/betacaroteno foram positivamente correlacionadas a duas das medidas de gordura corporal, sugerindo que quanto maior a gordura corporal, menor a quantidade de betacaroteno proporcional aos valores de CT e LDL. Moor de Burgos et al. ${ }^{9}$ observaram que indivíduos obesos têm deficiência 
de vitaminas antioxidantes, o que corrobora, ao menos em parte, a observação do presente estudo em relação à concentração plasmática de vitamina $\mathrm{C}$ e quanto à proporcionalidade de betacaroteno aos lipídios circulantes.

Em uma primeira análise dos dados deste estudo, parece que o avanço da idade reduziu a concentração de glóbulos brancos. As alterações sociais que ocorrem com a idade podem causar estresse emocional e desregular o sistema imunológico do idoso. Pode haver prejuízo na atividade dos linfócitos com várias conseqüências negativas para o sistema imunológico ${ }^{30}$. Entretanto, quando os glóbulos brancos foram corrigidos pelo peso corporal, a associação positiva com a idade desapareceu e a concentração de linfócitos não foi associada à idade, porém, não se mediu a sua atividade. Parece que no caso destas mulheres, a gordura corporal foi mais importante para determinar a associação ao sistema imunológico do que a idade. Ainda, elas não eram idosas, momento em que a depressão do sistema imunológico se torna mais evidente.

\section{O N CLUS Ã O}

Em suma, dois meses de um programa de reeducação de hábitos parecem ter reduzido os fatores de risco associados às DCNT, mas não interferiram nos marcadores do sistema imunológico destas mulheres. Todavia, o aumento da gordura corporal foi positivamente associado à piora dos marcadores do sistema imunológico, das vitaminas e do perfil lipídico, sugerindo que pode ser necessária atenção com o aporte de antioxidantes em indivíduos obesos que iniciem a prática de atividades físicas. Futuros estudos com grupo controle ao exercício físico, maior número de voluntários e um processo inflamatório crônico bem estabelecido, poderá auxiliar a esclarecer estas interações.

\section{$C O L A B O R A C ̧ A ̃ O$}

C.A. FETT escreveu o projeto e a versão final do manuscrito. W.C.R. FETT foi responsável pela apli- cação dos protocolos de avaliação, de atividade física, pela coordenação das reuniões semanais sobre reeducação de hábitos e pela análise estatística. J.G. PADOVANI foi responsável pela dosagem do zinco, pela análise dos métodos de laboratório e pela revisão da versão final do manuscrito. J.S. MARCHINI orientou e corrigiu desde o projeto até a versão final do manuscrito.

\section{REFERÊ N CIAS}

1. lyengar GV, Nair PP. Global outlook on nutrition and the environment: meeting the challenges of the next millennium. Sci Total Environ. 2000; 249 (1-3):331-46.

2. Al-Saleh I, El-Doush I, Billedo G, Mohamed Gel-D, Yosef $G$. Status of selenium, vitamin $E$, and vitamin A among Saudi adults: potential links with common endemic diseases. J Environ Pathol Toxicol Oncol. 2007; 26(3):221-43.

3. Apor P, Radi A. Physical exercise, oxidative stress and damage. Orv Hetil. 2006; 147(22):1025-31.

4. Ozata M, Mergen M, Oktenli C, Aydin A, Sanisoglu SY, Bolu $\mathrm{E}$, et al. Increased oxidative stress and hypozincemia in male obesity. Clin Biochem. 2002; 35(8):627-31.

5. Shim WS, Kim HJ, Kang ES, Ahn CW, Lim SK, Lee $\mathrm{HC}$, et al. The association of total and differential white blood cell counts with metabolic syndrome in type 2 diabetic patients. Diabetes Res Clin Pract. 2006; 73(3):284-91.

6. Couillard C, Pomerleau S, Ruel G, Archer WR, Bergeron J, Couture $\mathrm{P}$, et al. Associations between hypertriglyceridemia, dietary fat intake, oxidative stress, and endothelial activation in men. Nutrition. 2006; 22(6):600-8.

7. Fett CA, Fett WCR. Incidência de disbiose em fundistas. Rev Min Ed Fis. 2003; 11(1):25-36.

8. Jackson AS, Pollock ML, Ward A. Generalized equations for predicting body density of women. Med Sci Sports Exerc. 1980; 12(3):175-81.

9. Moor de Burgos A, Wartanowicz M, Ziemlanski S. Blood vitamin and lipid levels in overweight and obese women. Eur J Clin Nutr. 1992; 46(11): 803-8.

10. Segal $K R$, van Loan $M$, Fitzerald $P I$, Hodgdon JA, van Itallie TB. Lean body mass estimation by bioelectrical impedance analyses: a four-site cross-validation study. Am J Clin Nutr. 1988; 47(1):7-14.

11. Hospital das Clínicas da Faculdade de Medicina de Ribeirão Preto da Universidade de São Paulo. 
Procedimento técnicos dos laboratórios do HCRP. Ribeirão Preto: USP; 2000.

12. World Health Organization. Physical status: the use and interpretation of anthropometrics. Report of a World Health Organ Expert Committee. World Health Organ Tech Rep Ser. 1995; 854:1-452.

13. Han TS, van Leer EM, Seidell JC, Lean ME. Waist circumference action levels in identification o cardiovascular risk factors: prevalence study in a random sample. BMJ. 1995; 311(7017):1401-5.

14. Shidfar F, Baghai N, Keshavarz A, Ameri A, Shidfar S. Comparison of plasma and leukocyte vitamin C status between asthmatic and healthy subjects. East Mediterr Health J. 2005; 11(1-2):87-95.

15. Mafra D, Cozzolino SMF. Importância do zinco na nutrição humana. Rev Nutr. 2004; 17(1):79-87.

16. Wallstrom P, Wirfalt E, Lahmann PH, Gullberg B, Janzon L, Berglund G. Serum concentrations of beta-carotene and alpha-tocopherol are associated with diet, smoking, and general and central adiposity. Am J Clin Nutr. 2001; 73(4):777-85.

17. Czernichow S, Bertrais S, Oppert JM, Galan P, Blacher J, Ducimetiere $\mathrm{P}$, et al. Body composition and fat repartition in relation to structure and function of large arteries in middle-aged adults (the SU.VI.MAX study). Int J Obes. 2005; 29(7):82632.

18. Rabovsky A, Eich JCN. Measurement of plasma antioxidant reserve after supplementation with various antioxidants in healthy subjects. Clin Chim Acta. 2006; 371(1-2):55-60.

19. Wintergerst ES, Maggini S, Hornig DH. Immune-enhancing role of vitamin $\mathrm{C}$ and zinc and effect on clinical conditions. Ann Nutr Metab. 2006; 50(2):85-94.

20. Martinez-Abundis E, Pascoe-Gonzalez S, Gonzalez-Ortiz M, Mora-Martinez JM, Cabrera-Pivaral CE. Effect of oral administration of ascorbic acid on insulin sensitivity and lipid profile in obese individuals. Rev Invest Clin. 2001; 53(6):505-10.

21. Benabdeslam H, Abidi H, Garcia I, Bellon G, Gilly $R$, Revol A. Lipid peroxidation and antioxidant defenses in cystic fibrosis patients. Clin Chem Lab Med. 1999; 37(5):511-6.
22. Foksinski $M$, Gackowski D, Rozalski R, Siomek A, Guz J, Szpila A, et al. Effects of basal level of antioxidants on oxidative DNA damage in humans. Eur J Nutr. 2007; 46(3):174-80.

23. Kang DH, Nakagawa T. Uric acid and chronic renal disease: possible implication of hyperuricemia on progression of renal disease. Semin Nephrol. 2005; 25(1):43-9.

24. Waring WS, McKnight JA, Webb DJ, Maxwell SRJ. Uric acid restores endothelial function in patients with type 1 diabetes and regular smokers. Diabetes. 2006; 55(11):3127-32.

25. Chen MD, Lin PY, Lin WH. Investigation of the relationships between zinc and obesity. Gaoxiong Yi Xue Ke Xue Za Zhi. 1991; 7(12):628-34.

26. Pavao ML, Figueiredo T, Santos $V$, Lopes PA, Ferin $\mathrm{R}$, Santos MC, et al. Whole blood glutathione peroxidase and erythrocyte superoxide dismutase activities, serum trace elements (Se, Cu, Zn) and cardiovascular risk factors in subjects from the city of Ponta Delgada, Island of San Miguel, The Azores Archipelago, Portugal. Biomarkers. 2006; 11(5): 460-71.

27. Hughes S, Samman S. The effect of zinc supplementation in humans on plasma lipids, antioxidant status and thrombogenesis. J Am Coll Nutr. 2006; 25(4):285-91.

28. Juhan I, Gabrielli M, Jouve R, Calas MF, DurandDessemon F, Vague J. A study of platelet abnormalities in obese subjects (author's transl). Diabete Metab. 1980; 6(1):17-24.

29. Elbatarny HS, Netherton SJ, Ovens JD, Ferguson $\mathrm{AV}$, Maurice DH. Adiponectin, ghrelin, and leptin differentially influence human platelet and human vascular endothelial cell functions: implication in obesity-associated cardiovascular diseases. Eur J Pharmacol. 2007; 558(1-3):7-13.

30. Guidi L, Tricerri A, Frasca D, Vangeli M, Errani AR, Bartoloni C. Psychoneuroimmunology and aging. Gerontology. 1998; 44(5):247-61.

Recebido em: 10/5/2007

Versão final reapresentada em: 24/6/2008 Aprovado em: 18/8/2008 